\title{
Cooperation-based Clustering for Profit-maximizing Organizational Design
}

Christophe G. Giraud-Carrier

cgc@cs.byu.edu

Kevin Seppi

Brigham Young University, kseppi@byu.edu

Nghia Tran

Sean C. Warnick

Follow this and additional works at: https://scholarsarchive.byu.edu/facpub

Part of the Computer Sciences Commons

\section{Original Publication Citation}

Tran, N., Giraud-Carrier, C., Seppi, K. and Warnick, S. "Cooperation-based Clustering for Profitmaximizing Organizational Design." In Proceedings of the International Joint Conference on Neural Networks (IJCNN'6), pp. 3479-3483, Vancouver, B.C.

\section{BYU ScholarsArchive Citation}

Giraud-Carrier, Christophe G.; Seppi, Kevin; Tran, Nghia; and Warnick, Sean C., "Cooperation-based Clustering for Profit-maximizing Organizational Design" (2006). Faculty Publications. 982.

https://scholarsarchive.byu.edu/facpub/982 


\title{
Cooperation-based Clustering for Profit-maximizing Organizational Design
}

\author{
Nghia Tran, Christophe Giraud-Carrier, Kevin Seppi, and Sean Warnick, Member, IEEE
}

\begin{abstract}
This paper shows how the notion of value of cooperation, a measure of the percentage of a firm's profits due strictly to the cooperative effects among the goods it sells, can be used to analyze the relative economic advantage afforded by various organizational structures. The value of cooperation is computed from transactions data by solving a regression problem to fit the parameters of the consumer demand function, and then simulating the resulting profit-maximizing dynamic system under various organizational structures. A hierarchical agglomerative clustering algorithm can be applied to reveal the optimal organizational substructure.
\end{abstract}

\section{INTRODUCTION}

Analyzing the impact of organizational structure on the performance of profit-maximizing organizations is a difficult task for business managers. Yet, informed design decisions are essential to long-term profitability. While these decisions are often ad hoc, today's large volumes of data make more systematic analyses possible. The key concept in such analyses is that of the value of cooperation (VC) experienced by a firm, which captures the percentage of a firm's profits due strictly to the cooperative effects among the goods it sells [1].

Such a measure provides the scientific backing for sound organizational design decisions. For example, if a firm can identify which of its products have strong synergies with others, it can organize to ensure that decision makers of related products work closely together. This may include physically co-locating entities where interaction adds strong value to the organization, or it may result in decentralization when cooperation adds little value. Similarly, if a firm identifies pieces of its business that add little cooperative benefit to the organization as a whole, it may consider selling off these subunits. A subunit with a healthy balance sheet may sell for a high price without adversely affecting the firm's value of cooperation. On the other hand, the firm may pursue a different strategy of retaining its decoupled subunits but use the value of cooperation to identify an acquisition that strongly couples their mutual benefit. Thus, divisions of a firm that are quite independent may be cooperatively coupled through the acquisition of another business with the right cooperative effects. For example, a firm with two distinct independent divisions that have no value of cooperation may acquire another business unit that not only adds value of cooperation with each division, but does so in a way that the total business becomes tightly integrated. Moreover, the firm

All authors are with the Department of Computer Science, Brigham Young University, Provo, UT 84602, USA (phone: 801-422-3027; fax: 801422-0169; email: \{tcnghia,cgc,kseppi,sean\}@cs.byu.edu). may identify an acquisition candidate that is struggling on its own, and thus is inexpensive, but brings the right cooperative effects to the organization to offset the risk of acquiring a struggling business. The value of cooperation thus becomes the lens through which a firm can better identify valuable opportunities in the market environment, or costly "baggage" in its own organizational structure.

In this paper, we discuss a value of cooperation inspired by the theory of industrial organization and coalition games [2], [3], [4], [5], [6]. Essentially, the profit maximizing dynamics of a given organizational structure define the value function for a particular coalition game. Researchers in [7] describe how the impact of a proposed merger can be computed by evaluating the post-merger equilibrium prices. They consider common functional forms of demand functions and indicate how to conduct the merger simulation in each case. Our value of cooperation is computed through a kind of "reverse" merger simulation that explores the impact of splitting the firm into its constituent economic units to determine the value it is realizing by unifying the objectives of these basic units. We are then able to use this measure to drive a hierarchical agglomerative clustering algorithm in order to reveal the organizational substructure defined naturally from the cooperative effects of the global product network.

\section{The VAlue of COOPERATion}

Consider a market, $\mathcal{M}$, selling $N$ products. A firm, $F$, is a subset of the $N$ products in the market, $F \in 2^{\mathcal{M}}$. This implies that the firm controls the production and distribution of the products assigned to it. Furthermore, we consider a Bertrand market model, so that each firm may set the prices of the products assigned to it. We assume that the products of the market are partitioned between $m$ firms (i.e., no two firms control the same product) and that every firm in the market is a profit maximizing entity. Under the standard assumption that market dynamics are stabilizing, we expect price perturbations to re-equilibriate, which means that we may simply consider the profits of firms at equilibrium. These profits, in turn, define a payoff function reminiscent of those used to define coalition games.

Let $v\left(F_{i}\right)=\left.\pi_{i}\right|_{p=p_{e q}}$ be the payoff or profit of firm $i$ at the market equilibrium prices $p_{e q}$. In this way the firm may be thought of as a coalition of $n_{i}$ players in an $N$-player cooperative game. Each player is a one-product company that completely manages the production, distribution, and pricing decisions for its product. The firm, then, is a confederacy of these one-product companies that work together to maximize their combined profits or payoffs. 
The theory of coalition games studies the behavior of such coalitions once the payoff function is defined for every possible coalition. The idea is that any given coalition $F_{i}$ yields a well-defined payoff $v\left(F_{i}\right)$, and then a number of questions can be explored regarding how to distribute the payoff among the members of the coalition, etc.

Our situation is different, however, because the payoff to a given firm does not depend only on the products it controls, but also on the market structure of the products outside the firm. For example, consider a 10-product market and a three product firm in the market. The payoff to the firm does not depend only on the prices of the three products it controls, but also on the prices of the other seven products. The profitmaximizing equilibrium prices of these other seven products, however, may be set differently depending on whether they belong to a single firm or whether they are controlled by seven different firms. Thus, the payoff to the three-product firm depends on the entire market structure.

Coalition game theory addresses such situations by considering partition systems and restricted games. For our purposes, it is sufficient to partition the $N$ products of $\mathcal{M}$ into $m$ firms and assume that this structure is fixed outside of the particular firm that we are studying. This enables us to work with a well-defined payoff function induced by the profitmaximizing dynamics of firms within the market without eliminating the multiple-coalition (i.e., multiple firm) cases of interest.

To quantify the value of organizing a group of oneproduct companies into a single firm, we need to compare the profits the firm receives if it sets its prices as if each of its products were independent companies with those it realizes by fully capitalizing on cooperation between the products. More precisely, let $p_{e q}$ be the profit-maximizing equilibrium prices for the given market structure. In contrast, consider the new profit maximizing equilibrium prices achieved without cooperation if $F_{i}$ were divided into its constituent oneproduct companies and each independently optimized their prices. Let this second set of equilibrium prices serve as a basis for comparison, or reference, and be denoted $p_{\text {ref }}$. We can then define the following measure.

Definition 1: The Value of Cooperation (VC) of a firm $F_{i}$ in market $\mathcal{M}$ with structure $S=F_{1}, F_{2}, \ldots, F_{m}$ is given by:

$$
\mathrm{VC}_{\text {ref }}\left(F_{i}, S\right)=\left.\pi_{i}\right|_{p_{\text {eq }}}-\left.\pi_{i}\right|_{p_{\text {ref }}}
$$

This VC measure captures precisely the value realized by the firm due to cooperation within its organization. Note that the VC measure is always non-negative since the cooperating firm can always recover at least the non-cooperating, or reference, profits by simply setting the prices it controls in $p_{\text {eq }}$ to those of $p_{\text {ref }}$.

As defined, the VC measure carries units of dollars and reflects a kind of absolute dollar-value of cooperation within the firm, thus making comparisons difficult. We, therefore, define a "relative" Value of Cooperation by normalizing $\mathrm{VC}_{\text {ref }}$ by the equilibrium profits as follows.

Definition 2: The Relative Value of Cooperation (RVC) of a firm $F_{i}$ in market $\mathcal{M}$ with structure $S=F_{1}, F_{2}, \ldots, F_{m}$ is given by:

$$
\mathrm{RVC}_{\text {ref }}\left(F_{i}, S\right)=\frac{\left.\pi_{i}\right|_{p_{\text {eq }}}-\left.\pi_{i}\right|_{p_{\text {ref }}}}{\left.\pi_{i}\right|_{p_{\text {eq }}}}
$$

This RVC measure is naturally interpreted as the percentage of profits due to cooperation within the organization. It is bounded between zero and one, and enables direct comparison among firms of different sizes.

By simply replacing the equilibrium and reference prices in the above definitions with the equilibriated profitmaximizing prices of the market structures being compared, one can easily use the thus modified VC and RVC to analyze the relative values of different organizational structures within a single firm. This is a natural application of the above framework, where the market is a firm and the firms are its organizational divisions.

\section{A Simple EXAMPLE}

To illustrate the use of $\mathrm{VC}$ and RVC, let us consider a simple one-firm market with only two products with linear demand given by:

$$
\left[\begin{array}{l}
q_{1}(t) \\
q_{2}(t)
\end{array}\right]=\left[\begin{array}{cc}
-3.5 & -1 \\
-3 & -2
\end{array}\right]\left[\begin{array}{l}
p_{1}(t) \\
p_{2}(t)
\end{array}\right]+\left[\begin{array}{l}
100 \\
100
\end{array}\right]
$$

Suppose that the unit production costs of each product are $c_{1}=c_{2}=10$. If we consider a firm structure where each product is produced by an independent division, the profit function for each division becomes:

$$
\begin{aligned}
\pi_{1}(t) & =q_{1}(t)\left(p_{1}(t)-c_{1}\right) \\
& =-3.5 p_{1}^{2}-p_{1} p_{2}+135 p_{1}+10 p_{2}-1000 \\
\pi_{2}(t) & =q_{2}(t)\left(p_{2}(t)-c_{2}\right) \\
& =-2 p_{2}^{2}-3 p_{1} p_{2}+30 p_{1}+120 p_{2}-1000
\end{aligned}
$$

Taking the partial derivatives of each profit function with respect to the appropriate pricing variable, we get the profitmaximizing market dynamics:

$$
\left[\begin{array}{l}
\frac{d p_{1}(t)}{d t} \\
\frac{d p_{2}(t)}{d t}
\end{array}\right]=\left[\begin{array}{ll}
-7 & -1 \\
-3 & -4
\end{array}\right]\left[\begin{array}{l}
p_{1}(t) \\
p_{2}(t)
\end{array}\right]+\left[\begin{array}{l}
135 \\
120
\end{array}\right]
$$

These two-department dynamics drive an initial pricing vector to the following profit-maximizing equilibrium:

$$
p_{\text {ref }}=\left[\begin{array}{l}
16.8 \\
17.4
\end{array}\right]
$$

and associated equilibriated profits: $\pi_{1}=161.84$ and $\pi_{2}=$ 109.52 .

Now, consider a firm structure where both products are controlled by the same division. In this case, the firm's profit function becomes:

$$
\begin{aligned}
\pi(t) & =q_{1}(t)\left(p_{1}(t)-c_{1}\right)+q_{2}(t)\left(p_{2}(t)-c_{2}\right) \\
& =-3.5 p_{1}^{2}+165 p_{1}-4 p_{1} p_{2}+130 p_{2}-2 p_{2}^{2}-2000
\end{aligned}
$$


With this structure, the division adjusts the prices of both products to optimize the same objective. The new dynamics become:

$$
\left[\begin{array}{l}
\frac{d p_{1}(t)}{d t} \\
\frac{d p_{2}(t)}{d t}
\end{array}\right]=\left[\begin{array}{ll}
-7 & -4 \\
-4 & -4
\end{array}\right]\left[\begin{array}{l}
p_{1}(t) \\
p_{2}(t)
\end{array}\right]+\left[\begin{array}{l}
165 \\
130
\end{array}\right]
$$

These single-division dynamics drive an initial pricing vector to the following profit-maximizing equilibrium:

$$
p_{e q}=\left[\begin{array}{l}
11.67 \\
20.83
\end{array}\right]
$$

and associated equilibriated profit: $\pi_{e q}=316.667$.

The values of cooperation in this example thus become: $V C=\pi_{e q}-\left(\pi_{1}+\pi_{2}\right)=45.3067$ and $R V C=0.1431$. These suggest that in this market, just under $15 \%$ of the profits of the two-product firm are the direct result of its inter-division cooperation.

\section{VC-BASED CLUSTERING}

Equipped with the value of cooperation as a measure of productive interaction between products, a firm may consider its internal organization and cluster its products to maximize the value of cooperation, indicating which decision makers in the organization should work together in setting prices, and which can work more independently. Knowing where cooperative value resides within an organization is the critical first step in exploiting it.

We begin by describing how one may compute a firm's value of cooperation from data. The algorithmic process involves the following three steps:

1) Fit demand model from transactions data. This can often be accomplished through standard regression techniques. To obtain good estimates of the model parameters, however, it is important that the data be sufficiently "exciting." This can often be a challenge if prices have remained relatively constant, or have only been changed in very structured ways (e.g., $20 \%$ off everything sales).

2) Build corresponding profit-maximizing dynamic system. This step involves constructing the firm's profit function for each product in its offering. Importantly, this assumes knowledge of the total handling costs associated with selling each product, which information may often have to be estimated at best. Moreover, our initial results assume that this cost structure does not change with organization structure, i.e., although economies of scale associated with selling more of a product can be easily accommodated, no cost benefit across product lines is built into the existing model. ${ }^{1}$

3) Compute equilibrium for given organizational structures. With different assumptions about which products group together, the profit maximizing dynamics

\footnotetext{
${ }^{1}$ This limitation is an important focus of future work since synergistic costs can play as important a role in total cooperation as synergistic sales. Nevertheless, the current work demonstrating effects of synergistic sales demonstrates the key idea underlying cooperation-based analysis.
}

are altered yielding a new equilibrium point for the system. These equilibrium profits generate a new set of equilibrium profits, and it is through a comparison of equilibrium profits that the value of cooperation is calculated. Note that depending on the size of the problem or the strength of nonlinearities in the dynamics, these equilibria may often be computed analytically, or through simulation.

A natural extension of the above algorithm would be to automate step 3 in such way that the organizational structure resulting in the largest value of cooperation is found. In other words, one is interested in finding the $k$ clusters of $n$ products for which the total value of cooperation is maximized for all $k$ from 1 to $n$. We propose to do this through the use of the value of cooperation within a hierarchical agglomerative clustering framework [8], [9].

VC-based hierarchical agglomerative clustering starts from the reference structure, where all products are assumed to act independently. Then, the two products which exhibit the strongest value of cooperation are merged, and the process is repeated, decreasing the total number of clusters by one until all of the firm's products are finally merged into a single organizational structure. The hierarchical nesting adds a natural constraint to the problem, which yields a product hierarchy with a clear organizational interpretation.

The real novelty of our approach comes from its use of the profit-maximizing dynamics to define the resulting clusters. A typical approach to economic-driven clustering may compute the same demand function from data, expand it in a Taylor series around the market equilibrium, and then consider the first order terms as defining a graph over products, i.e., the product network. Various approaches to clustering this graph might then be considered. The approach discussed here, however, is a radical departure from such approaches by using the demand function to characterize a dynamic system, and then allowing this dynamic system to define clusters over the product network.

\section{EXPERIMENTS}

The use of VC-based hierarchical agglomerative clustering is best highlighted through an example. In this simple example, a firm managing 15 products is considered. The demand function is taken to have the form:

$$
q=A p+B
$$

where $q \in \mathbb{R}^{n}, p \in \mathbb{R}^{n}$, and $q_{i}, p_{i} \geq 0$ for $i=1, \ldots, n$. This linear structure may have been fit directly from data, or it may be the result of linearizing another demand function around a nominal set of prices.

Considering the reference structure where every product sets its price independently to maximize its own profit, each constituent product system has a profit function given by:

$$
\pi_{i}=q_{i}(p)\left(p_{i}-c_{i}\right)
$$

where $c_{i} \in \mathbb{R}^{+}$is the marginal cost of the $i^{t h}$ product. Note that a fixed cost could be added to the expression without affecting the results. 
Figure 1 illustrates the matrix $A$ in the demand function, both in tabular and graphical form.

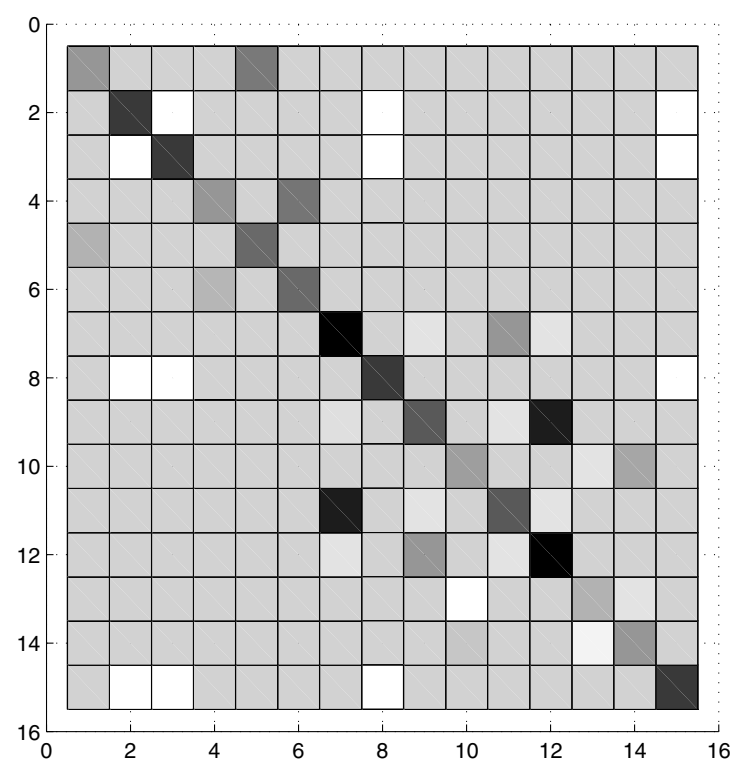

Fig. 1. The $A$ Matrix of the Demand Function

The vector $B$ in the demand function is given by:

$$
B=\begin{array}{ccccc}
{[1130} & 330 & 330 & 1130 & 1030 \ldots \\
1030 & 1930 & 330 & 2130 & 1125 \ldots \\
2130 & 1930 & -150 & 300 & 330]^{T}
\end{array}
$$

and the cost vector is given by:

$$
C=\begin{array}{lllll}
{[110} & 130 & 130 & 110 & 110 \ldots \\
110 & 110 & 130 & 110 & 120 \ldots \\
110 & 110 & 120 & 120 & 130]^{T}
\end{array}
$$

Note that the relative strength of the own-price elasticities of various products is visible in the strongly diagonal structure of matrix $A$. In spite of this feature, Figure 2 demonstrates that over $50 \%$ of the firm's profits result strictly from the cooperative effects between products.

The relative value of cooperation increases sharply as the first few products are grouped into their respective clusters. Once a critical clustering is achieved, however, no improvement in the value of cooperation is observed through subsequent centralization. This indicates that these sets of products are fairly independent, decoupled with respect to the market demand function. Cooperation-based clustering identifies these groups, even when they are not apparent from the market demand function directly.

The result of the cooperation-based clustering is shown in Figure 3. Each row indicates a set of clusters, beginning with 15 single-product clusters and ending with a single cluster of all the products. An interesting aspect of this clustering is apparent in the analysis of this figure with the RVC plot. We note that once the products have been grouped into five

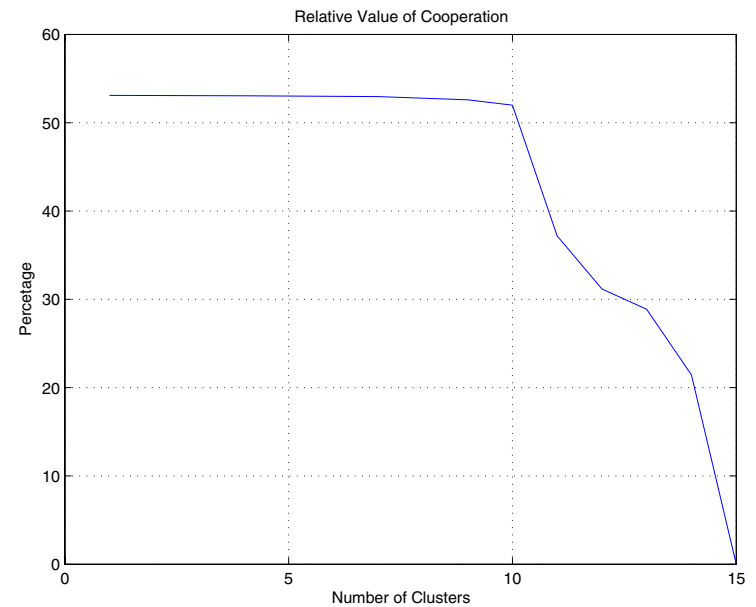

Fig. 2. RVC vs. Product Groupings

clusters, no more value of cooperation is derived through further clustering. This indicates that the firm is operating at the intersection of five rather independent markets, a fact that is not readily apparent from inspection of the demand function (see Figure 1).

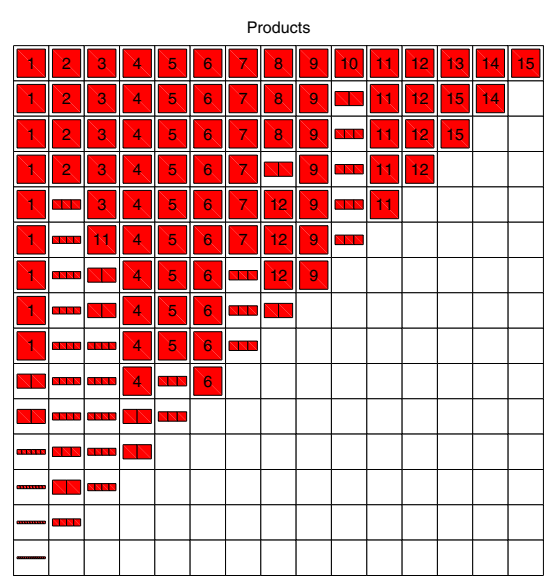

Fig. 3. CV-based Hierarchical Agglomerative Clustering of a 15 Product Firm

\section{CONCLUSION}

This paper explores the notion of value of cooperation, which measures the percentage of a firm's profits due strictly to the cooperative effects among the goods it sells. The idea is to assume profit-maximizing dynamics among firms within the market and compare equilibrium profits in two different scenarios. The first scenario considers the firm as it is, a single economic entity with a unified objective and exhibiting full cooperation among its various products. The second scenario considers splitting the firm into its constituent economic units and computing market equilibrium prices if 
these units were to fail to cooperate and acted completely independently out of self interest. The difference between the cooperative profits of the first scenario and the aggregate profits of the independent units of the second scenario defines what we call the value of cooperation of the firm in its current market environment. We have illustrated the use of the value of cooperation for product clustering in the context of optimal organizational design.

It is instructive to contrast the value of cooperation with other measures used to characterize cooperative games. Hart and Mas-Colell defined a measure, called the potential, $P$, that computes the expected normalized worth of the game, i.e., the per-capita potential, $P / N$, equals the average percapita worth $(1 / m) \sum_{i}\left(\pi_{i}\right) /\left(\left|F_{i}\right|\right)$. Given a market structure, this measure characterizes the expected profit of an averagesized firm (where size is measured with respect to the number of products the firm controls) in the market, even if such a firm does not actually exist. Moreover, the potential has been connected to another measure, called the Shapley value, $\Phi_{j}$, which yields the marginal contribution of each product in the market. This measure characterizes how the payoff of a coalition should be divided between members of the team. In both cases, the potential and Shapley values do not suggest anything about the intrinsic benefit of forming coalitions in the first place. The value of cooperation, on the other hand, captures the natural significance for organizing production into multi-product firms. Nevertheless, these measures do not yield any information about how the profit of a firm should be efficiently invested into each of the firm's constituent production lines. Thus, the measures are inherently different from the potential or Shapley value of the coalition game that focus more on the value of a member of a coalition to the group rather than the value of the coalition as a whole.

Future work will concretely establish the relationship between the value of cooperation and market power and indicate how to compute approximations to these metrics from readily available market data.

\section{ACKNOWLEDGMENT}

We would like to thank David Sims for his thoughtful discussions on the nature of economic systems and the meaning of cooperative value.

\section{REFERENCES}

[1] N. Tran, D. West, C. Giraud-Carrier, K. Seppi, S. Warnick, and R. Johnson, "The value of cooperation within a profit maximizing organization," in Proceedings of the Fourth International Conference on Computational Intelligence in Economics and Finance at the Eighth Joint Conference on Information Sciences, 2005, pp. 1017-1020.

[2] O. Shy, Industrial Organization: Theory and Applications. The MIT Press, 1996.

[3] J. Bilbao, Cooperative games on combinatorial structures. Kluwer Academic Publishers, Dordrecht, 2000.

[4] P. Morris, Introduction to Game Theory (Universitext). SpringerVerlag, 1994.

[5] L. Shapley, "A value for $n$-person games," in Contributions to the Theory of Games, Vol. II, ser. Annals of Mathematics Studies, H. Kuhn and A. Tucker, Eds. Princeton University Press, Princeton, NJ, 1953, vol. 28, pp. 307-317.

[6] S. Hart and A. Mas-Colell, "Potential, value, and consistency," Econometrica, vol. 57, no. 3, pp. 589-614, 1989.
[7] P. Crooke, L. Froeb, S. Tschantz, and G. Werden, "Effects of the assumed demand system on simulated postmerger equilibrium," U.S. Department of Justice - Antitrust Division, Tech. Rep. 97-3, 1997, available at $\mathrm{http}: / /$ ideas.repec.org/p/fth/usjuat/97-3.html.

[8] S. Johnson, "Hierarchical clustering schemes," Psychometrika, vol. 2, pp. 241-254, 1967.

[9] A. Jain and R. Dubes, Algorithms for Clustering Data. Prentice-Hall, Inc., 1988. 